\title{
Drug potency
}

Addiction Ontology

\section{Source}

Addiction Ontology

Definition: A quality of a drug that is the amount of a desired effect of the drug for a given dose and a given mode of administration.

Comment: Drugs may have different potencies for different drug effects. Effect of the drug on side-effects is not covered by this definition. If a drug has more than one effect that may be desired, it is necessary to specify what effect or effects are included in a given usage of the term.

This definition was imported from the Addiction Ontology (see https://addictovocab.org/). Comments and suggestions for improvements are welcome using the Qeios review system.

Definitions imported from the Addiction Ontology (AddictO) are what are known as 'ontological definitions'. See this article in Qeios for an explanation https://www.qeios.com/read/YGIF9B.

Ontological definitions can sometimes be hard to read. In those cases we also include an informal definition.

Definitions also often require elaboration to make it clear how they should be used and what they include. In those cases we include a comment.

Also, definitions sometimes require an explanation as to how they came about to help users understand how they relate to alternative definitions. In those cases we include a curator note. 